\title{
Geospace exploration project ERG
}

\author{
Yoshizumi Miyoshi 1*(D, Iku Shinohara², Takeshi Takashima², Kazushi Asamura², Nana Higashio², \\ Takefumi Mitani ${ }^{2}$, Satoshi Kasahara ${ }^{4}$, Shoichiro Yokota ${ }^{5}$, Yoichi Kazama ${ }^{6}$, Shiang-Yu Wang ${ }^{6}$, Sunny W. Y. Tam ${ }^{7}$, \\ Paul T. P. Ho ${ }^{6}$, Yoshiya Kasahara ${ }^{8}$, Yasumasa Kasaba ${ }^{9}$, Satoshi Yagitani ${ }^{8}$, Ayako Matsuoka ${ }^{2}$, Hirotsugu Kojima ${ }^{10}$, \\ Yuto Katoh ${ }^{9}$, Kazuo Shiokawa ${ }^{1}$ and Kanako Seki ${ }^{4}$
}

\begin{abstract}
The Exploration of energization and Radiation in Geospace (ERG) project explores the acceleration, transport, and loss of relativistic electrons in the radiation belts and the dynamics for geospace storms. This project consists of three research teams for satellite observation, ground-based network observation, and integrated data analysis/simulation. This synergetic approach is essential for obtaining a comprehensive understanding of the relativistic electron generation/loss processes of the radiation belts as well as geospace storms through cross-energy/cross-regional couplings, in which different plasma/particle populations and regions are strongly coupled with each other. This paper gives an overview of the ERG project and presents the initial results from the ERG (Arase) satellite.
\end{abstract}

Keywords: The ERG project, The ERG (Arase) satellite, The radiation belts, Geospace

\section{Introduction}

The radiation belts consist of energetic charged particle populations that are trapped in the inner magnetosphere. Associated with solar wind disturbances, the outer radiation belt electron population varies considerably. In particular, a decrease in the flux of relativistic electrons is observed during the main phase of geospace storms. The flux then recovers and often significantly increases during the recovery phase of storms (e.g., Nagai 1988; Miyoshi and Kataoka 2005). The identification of mechanisms that drive the flux variations of relativistic electrons in the radiation belts is fundamental and important problem in the solar-terrestrial physics.

There are several plasma and particle populations in the inner magnetosphere. The plasmasphere consists of cold/dense plasma populations, and the ionosphere is the source of the plasmaspheric plasma. The typical energy of the plasmaspheric plasma is less than $1 \mathrm{eV}$. The typical ring current and plasma sheet energies range from a few $\mathrm{keV}$ to about $100 \mathrm{keV}$, and these populations contribute to the ambient plasma pressure. The typical energies of the radiation belt particles range from a few hundred $\mathrm{keV}$

*Correspondence: miyoshi@isee.nagoya-u.ac.jp

${ }^{1}$ ISEE, Nagoya University, Nagoya 464-8601, Japan

Full list of author information is available at the end of the article to more than $10 \mathrm{MeV}$, which are the highest energy population in geospace. Thus, plasma and particle populations with a wide range of energies from a few $\mathrm{eV}$ to more than $10 \mathrm{MeV}$ coexist in the inner magnetosphere. Reviews on the inner magnetosphere can be found in several papers (e.g., Ebihara and Miyoshi 2011).

Two different mechanisms have been considered to cause the large flux enhancement of the outer radiation belt electrons. One is an external source process via quasi-adiabatic acceleration, which is the so-called radial diffusion. The other mechanism is an internal acceleration process. Figure 1 shows a diagram of the expected transportation and acceleration mechanisms as a function of the L-shell and energy. In the radial diffusion, i.e., the external source process (blue arrows), the electrons move earthward with increasing energy because of the conservation of the first two adiabatic invariants. The drift resonance with the MHD fast-mode waves is expected to cause the radial transportation of electrons, and the ultra-low frequency (ULF) geomagnetic pulsations of Pc4 and Pc5 are the primary waves that cause the resonance (e.g., Elkington et al. 1999). During the internal acceleration process (red arrows), sub-relativistic electrons are accelerated to $\mathrm{MeV}$ energies by whistler mode waves inside the radiation belts (e.g., Summers et al. 1998; Miyoshi et al. 2003; Thorne 2010). In this process, 


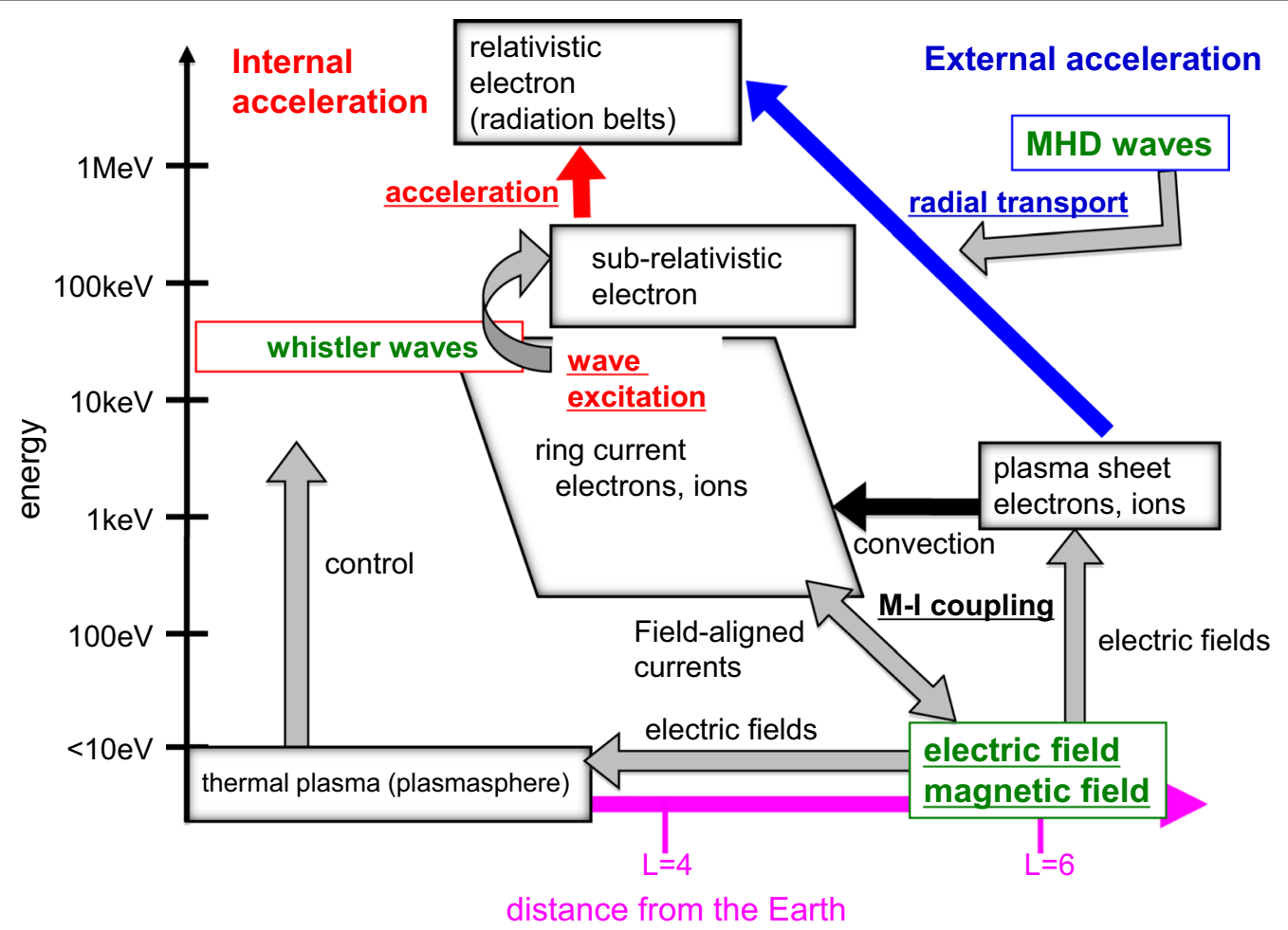

Fig. 1 Diagram of cross-energy coupling processes in the inner magnetosphere (modified from Miyoshi et al. 2012)

the whistler mode waves act as a mediating agent. The waves are generated through the plasma instability of hot electrons and subsequent nonlinear interactions (e.g., Omura et al. 2009), and the energy of the hot electrons transfers to the waves. The waves accelerate sub-relativistic electrons and enhance the relativistic electron flux. The cold plasma density population also contributes to this process because the cold plasma density controls the wave dispersion and resonance conditions. Thus, the cross-energy coupling of different energy plasmas and particle populations via wave-particle interactions plays an important role in the flux enhancements of the radiation belt electrons (Miyoshi et al. 2012, 2016).

There are debates on which external source process and internal acceleration process are important for the large flux enhancement of the outer radiation belt electrons. Phase space density profiles were derived from NASA/ Van Allen Probe observations (Mauk et al. 2013) in the October 2012 geospace storm, and it is identified for the first time that the internal acceleration process was dominant during the flux enhancement of $\mathrm{MeV}$ electrons (Reeves et al. 2013). It is expected that both the external source and internal acceleration processes are not exclusive, and the dominant processes that cause the large flux enhancements depend on the events (e.g., Reeves et al. 2003). So, long-term observations of the radiation belts are important to clarify how the external source and internal acceleration processes depend on different geomagnetic and solar wind conditions.

To understand the detailed acceleration and transportation processes, it is necessary to know all parameters that contribute to the accelerations. Electrons over wide energy ranges and electric and magnetic fields with wide frequency ranges are indispensable for understanding these processes. Observations of the ambient plasma density are also necessary. In addition, it is essential to simultaneously evaluate the loss processes in the radiation belts. Several processes contribute to the loss of the radiation belt electrons, escaping into the magnetopause, and the pitch angle scattering into the atmosphere because of the whistler mode waves and electromagnetic ion cyclotron (EMIC) wave-particle interactions (e.g., Turner et al. 2012).

Here, we describe the importance of multi-point observations in the inner magnetosphere. Several kinds of plasma waves play a key role for accelerations and loss of electrons in the radiation belt electrons. Whistler mode chorus waves accelerate relativistic electrons on the dawnside outside the plasmapause, whereas whistler mode hiss waves cause pitch angle scattering inside the plasmasphere. EMIC waves cause a strong scattering of $\mathrm{MeV}$ electrons (e.g., Miyoshi et al. 2008), which are 
mainly observed on the dusk and noon sides (e.g., Shprits et al. 2008a, b). These wave-particle interactions often occur simultaneously at different local times and radial distances.

Moreover, the causes and consequences of the processes are often observed at different locations. For example, the pitch angle scattering with whistler mode waves occurs in the magnetosphere, while the resultant precipitations are observed at ionospheric altitudes. In this example, it is possible to identify the causal relationship using conjugate observations between satellite and ground-based instruments. Thus, multi-point observations by multiple satellites and conjugate observations with ground-based observations are necessary for understanding the dynamics of the inner magnetosphere.

In addition to the observations, simulation studies are helpful for investigating the cause and consequence of phenomena and identify the relative role of each mechanism, because different physical processes work simultaneously and strongly couple with each other. Comprehensive simulations and modeling for the whole inner magnetosphere including different physical processes are necessary to identify the role of each process.

In order to elucidate the flux enhancement and loss processes of the relativistic electrons of the outer radiation belt as well as the dynamics of geospace storms through cross-energy and cross-regional couplings, the Exploration of energization and Radiation in Geospace (ERG) project has been organized. The following teams are involved in the ERG project: the satellite observation team, ground-based network observation team, and integrated data analysis/simulation team. The synergetic approach with these teams provides a comprehensive view of the radiation belts and inner magnetosphere for geospace storms. The main science questions of this project are as follows:

1. What are the dominant processes to cause the large flux enhancement of relativistic electrons of the outer radiation belt?

2. How do wave-particle interactions accelerate the relativistic electrons of the outer radiation belt?

3. What are the dominant loss processes for the relativistic electrons of the outer radiation belt?

In this paper, we describe an overview of the ERG project and show the initial observation results from the ERG (Arase) satellite.

\section{ERG (Arase) satellite \\ Overview}

The ERG (Arase) satellite was developed by the Institute of Space and Astronautical Science (ISAS)/Japan
Aerospace Exploration Agency (JAXA), and nine science instruments were developed at ISAS/JAXA as well as several universities and institutes in Japan and Taiwan. The satellite was successfully launched by the Epsilon launch vehicle from the Uchinoura Space Center of JAXA on December 20, 2016. Table 1 presents the specifications of the spacecraft, and Fig. 2 shows an image of the satellite in the radiation belts.

The ERG satellite is designed to be Sun oriented and spin stabilized with a rotation rate of $7.5 \mathrm{rpm}$. The apogee altitude is $\sim 32,000 \mathrm{~km}$, and the perigee altitude is $\sim 400 \mathrm{~km}$, so that the satellite explores the entire region of the radiation belts. The inclination angle of the satellite orbit is about $31^{\circ}$, and therefore, the satellite sometimes covers a wide $\mathrm{L}$-shell region up to about $10-11$ when the apogee altitudes are at large magnetic latitudes (MLATs). Details of the satellite system have been introduced by other papers (Takashima et al. 2018; Nakamura et al. 2018).

Figure 3 shows the orbit of the ERG satellite for four different seasons in the equatorial plane and meridional plane. The initial magnetic local time (MLT) of the apogee altitude was nine, and the apogee MLT then shifted from dawn to dusk through the night. Because the critical phase and commissioning phase operation took 3 months, regular observations with full operation of all the instruments started at 0600 MLT in March, 2017. The lines of the apogee progress in local time at a rate of approximately $260^{\circ}$ per year in the clockwise direction (looking down from the north).

Immediately after the beginning of regular observations in March 2017, the ERG satellite observed the inner magnetosphere near the magnetic equator at the heart of the outer radiation belt $(L=4-6)$ on the morning side (black curves). Because the generation of chorus waves is typically expected in this area (e.g., Shprits et al. 2008a, b), the science objectives on the morning side were the chorus wave-particle interactions.

The apogee MLT shifted to the night side during the summer of 2017 (blue curve) and then the dusk side

\section{Table 1 Outline of the ERG satellite}

\begin{tabular}{ll}
\hline Launch vehicle & Epsilon-2 \\
\hline Launch & December 20, 2016 \\
Weight $(\mathrm{kg})$ & $\sim 350$ \\
Size $(\mathrm{m})$ & $1.5 \times 1.5 \times 1.7$ \\
Orbit $(\mathrm{km})$ & $\sim 32,000$ (Apogee altitude), 400 (Perigee altitude) \\
Inclination angle $\left(^{\circ}\right)$ & 31 \\
Orbit period $(\mathrm{min})$ & $\sim 570$ \\
Science payload & Nine scientific instruments (see Table 2$)$ \\
Appendages & Two pairs of 5-m masts, two pairs of 30-m tip-to-tip \\
& $\quad$ wire antennas \\
\hline
\end{tabular}




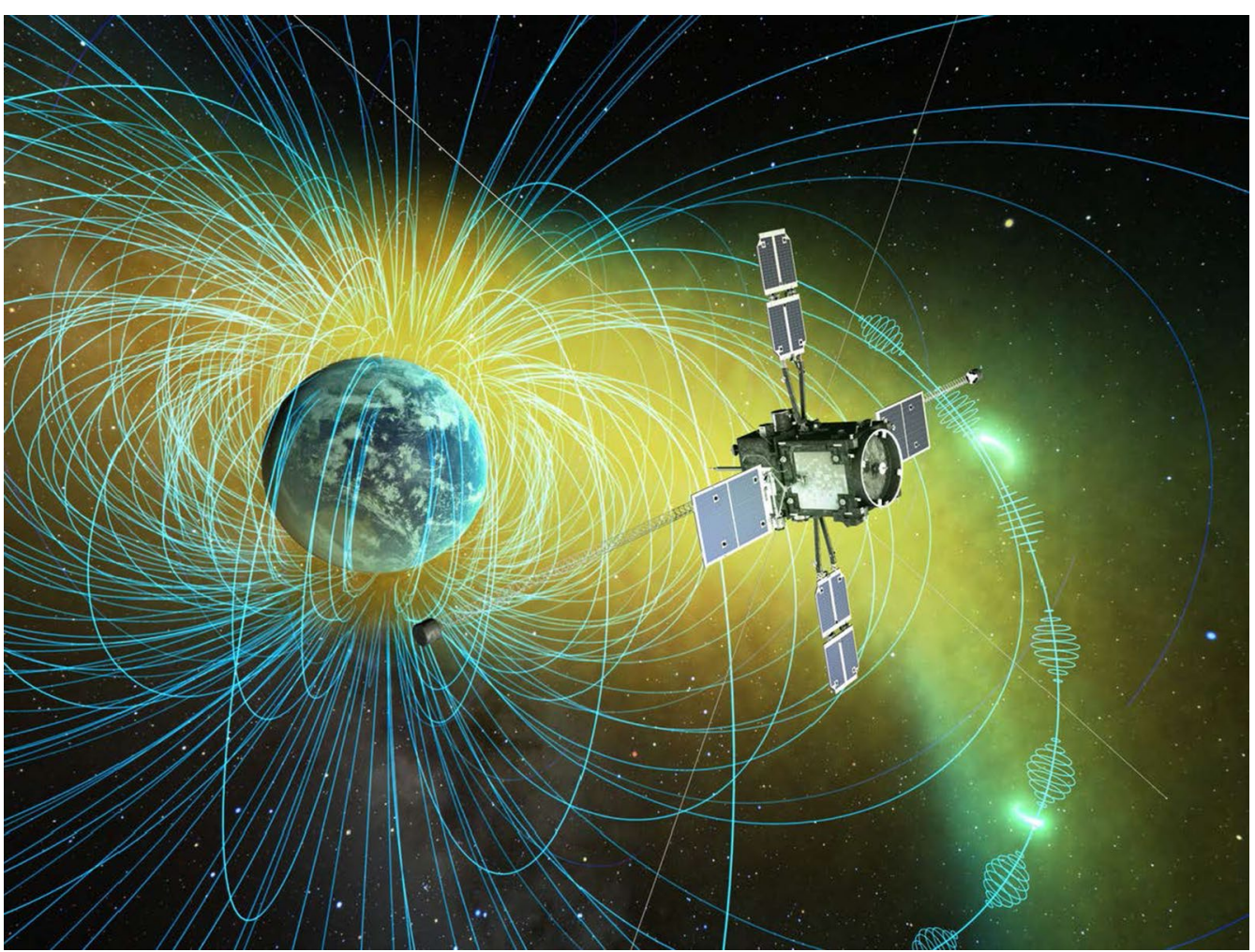

Fig. 2 Image of the ERG satellite observations in the geospace
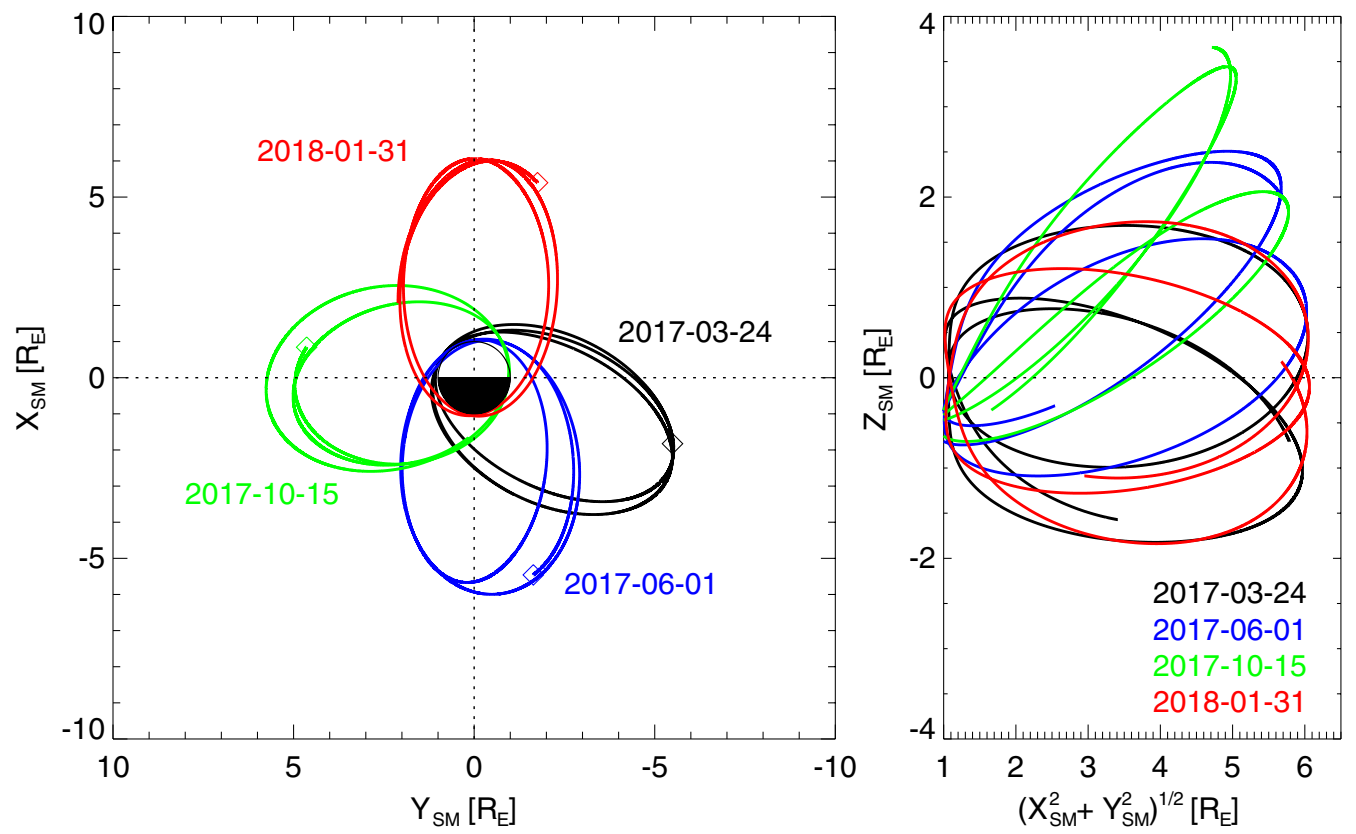

Fig. 3 Sample orbits of the ERG satellite. Black: March 24, 2017, Blue: June 1, 2017, Green: October 15, 2017, and Red: January 31, 2018 
during the fall of 2017 (green curve). During this period, the apogee MLATs were about $30^{\circ}-40^{\circ}$ and the ERG satellite covered a wider $L$-shell region up to $L \sim 9$ and often observed not only the plasma sheet and inner magnetosphere but also the plasma sheet boundary layer and tail lobes near the apogee.

Then, the apogee MLT moved to the noon side (red curve). The ERG satellite covered around the magnetic equator at the apogee altitudes. Because the EMIC waves are typically observed on the dusk and noon sides (e.g., Shprits et al. 2008a, b), the wave-particle interactions between the EMIC waves and ring current ions as well as the relativistic electrons in the outer radiation belt were the primary science objectives during this period.

\section{Science instruments}

Comprehensive observations of the inner magnetosphere for plasma/particles and fields/waves are important for understanding the role of cross-energy coupling for the dynamics of relativistic electrons as well as geospace storms. Nine instruments are installed on the ERG satellite, as shown in Fig. 4 and Table 2.

Figure 5 shows the energy and frequency coverages of these instruments. To measure electrons over a wide range of energies, including warm electrons, hot electrons in the plasma sheet and ring current, and sub-relativistic and relativistic electrons of the radiation belts, four instruments are installed: low-energy particle experiments-electron analyzer (LEP-e) (Kazama et al. 2017), medium-energy particle experiments-electron analyzer (MEP-e) (Kasahara et al. 2018a), high-energy electron

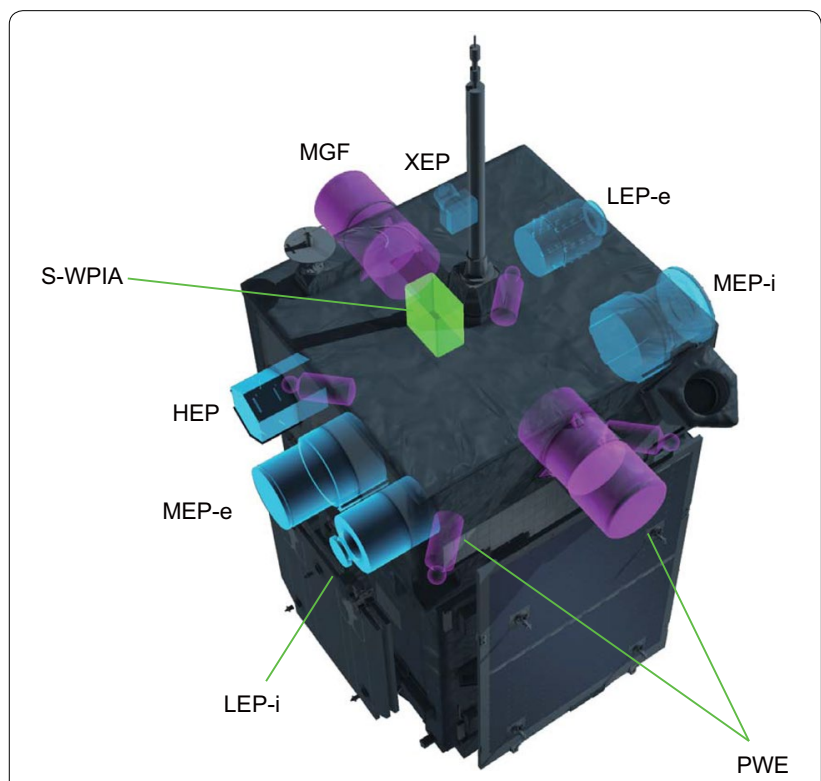

Fig. 4 Configuration of science instruments onboard the ERG satellite
Table 2 List of science instruments onboard ERG satellite

\begin{tabular}{|c|c|c|}
\hline Instrument & & Measurement \\
\hline LEP-e & $\begin{array}{c}\text { Low-energy particle experi- } \\
\text { ments-electron analyzer }\end{array}$ & 19 eV-20 keV (electron) \\
\hline MEP-e & $\begin{array}{l}\text { Medium-energy particle } \\
\text { experiments-electron } \\
\text { analyzer }\end{array}$ & 7-87 keV (electron) \\
\hline \multirow[t]{2}{*}{ HEP } & $\begin{array}{l}\text { High-energy electron } \\
\text { experiments }\end{array}$ & \\
\hline & $\begin{array}{l}\text { HEP-L } \\
\text { HEP-H }\end{array}$ & $\begin{array}{l}70 \mathrm{keV}-1 \mathrm{MeV} \text { (electron) } \\
0.7-2 \mathrm{MeV} \text { (electron) }\end{array}$ \\
\hline XEP & $\begin{array}{c}\text { Extremely high-energy } \\
\text { electron experiment }\end{array}$ & 400 keV-20 MeV (electron) \\
\hline LEP-i & $\begin{array}{c}\text { Low-energy particle experi- } \\
\text { ments-ion mass analyzer }\end{array}$ & $\begin{array}{r}10 \mathrm{eV} / \mathrm{q}-25 \mathrm{keV} / \mathrm{q} \text { (ions) } \\
\text { (mass discriminations) }\end{array}$ \\
\hline MEP-i & $\begin{array}{l}\text { Medium-energy particle } \\
\text { experiments-ion mass } \\
\text { analyzer }\end{array}$ & $\begin{array}{l}\text { 10-180 keV/q (ions) (mass } \\
\text { discriminations) }\end{array}$ \\
\hline \multirow[t]{5}{*}{ PWE } & Plasma wave experiment & \\
\hline & $\begin{array}{l}\text { Electric field detector } \\
\quad(\text { EFD) }\end{array}$ & $\begin{array}{l}\text { DC-512 Hz (electric field } \\
\text { waveform and spectrum) }\end{array}$ \\
\hline & $\begin{array}{l}\text { Onboard frequency } \\
\text { analyzer (OFA) }\end{array}$ & $\begin{array}{l}10 \mathrm{~Hz}-20 \mathrm{kHz} \text { (electric field } \\
\text { spectrum) } \\
1 \mathrm{~Hz}-20 \mathrm{kHz} \text { (magnetic field } \\
\text { spectrum) }\end{array}$ \\
\hline & Waveform capture (WFC) & $\begin{array}{l}10 \mathrm{~Hz}-20 \mathrm{kHz} \text { (electric field } \\
\text { waveform) } \\
1 \mathrm{~Hz}-20 \mathrm{kHz} \text { (magnetic field } \\
\text { waveform) }\end{array}$ \\
\hline & $\begin{array}{l}\text { High-frequency analyzer } \\
\text { (HFA) }\end{array}$ & $\begin{array}{l}10 \mathrm{kHz}-10 \mathrm{MHz} \text { (electric field } \\
\text { spectrum) } \\
10 \mathrm{kHz}-100 \mathrm{kHz} \text { (magnetic } \\
\text { field spectrum) }\end{array}$ \\
\hline MGF & Magnetic field experiment & $\begin{array}{l}\text { DC-256 Hz (magnetic field } \\
\text { waveform) }\end{array}$ \\
\hline S-WPIA & $\begin{array}{c}\text { Software-type wave-parti- } \\
\text { cle interaction analyzer }\end{array}$ & $\begin{array}{l}\text { Phase between waves and } \\
\text { particles }\end{array}$ \\
\hline
\end{tabular}

experiment (HEP) (Mitani et al. 2018), and extremely high-energy electron experiment (XEP). In parallel, to measure the warm ions and energetic ions of the plasma sheet and ring current with mass discrimination, two instruments are installed: low-energy particle experiments-ion analyzer (LEP-i) (Asamura et al. 2018) and medium-energy particle experiments-ion mass analyzer (MEP-i) (Yokota et al. 2017). The energy ranges of the instruments are designed to overlap each other to provide a continuous energy spectrum for various plasma/ particle populations in the inner magnetosphere.

From these instruments, the ERG satellite measures electrons with a wide range of energies, from $19 \mathrm{eV}$ to $20 \mathrm{MeV}$. Moreover, not only the pitch angle distributions but also the three-dimensional distribution function data are obtained up to $2 \mathrm{MeV}$ in each spin period from the LEP-e/MEP-e/HEP instruments. In the wave-particle interactions, low-energy electrons contribute to the 
a Energy Coverages: LEP-i, MEP-i, LEP-e, MEP-e, HEP, XEP

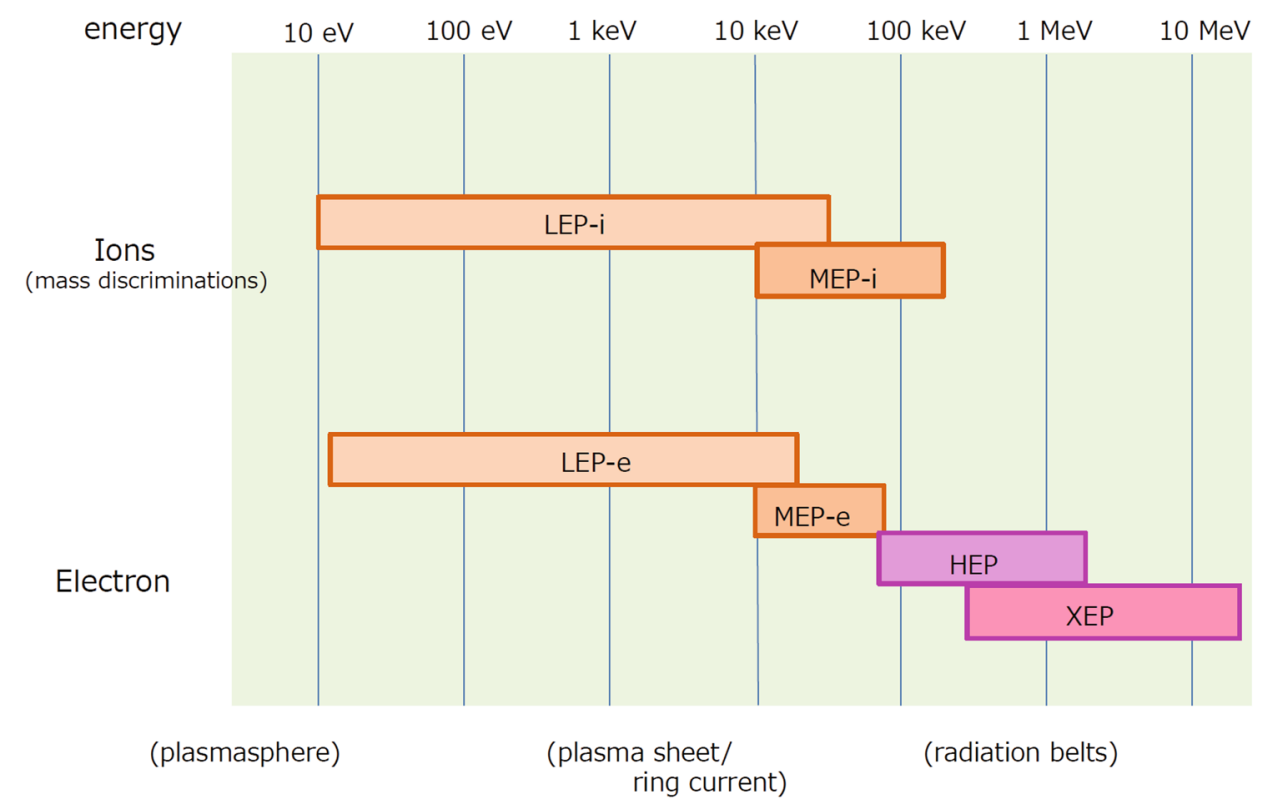

b Frequency Coverages: PWE, MGF

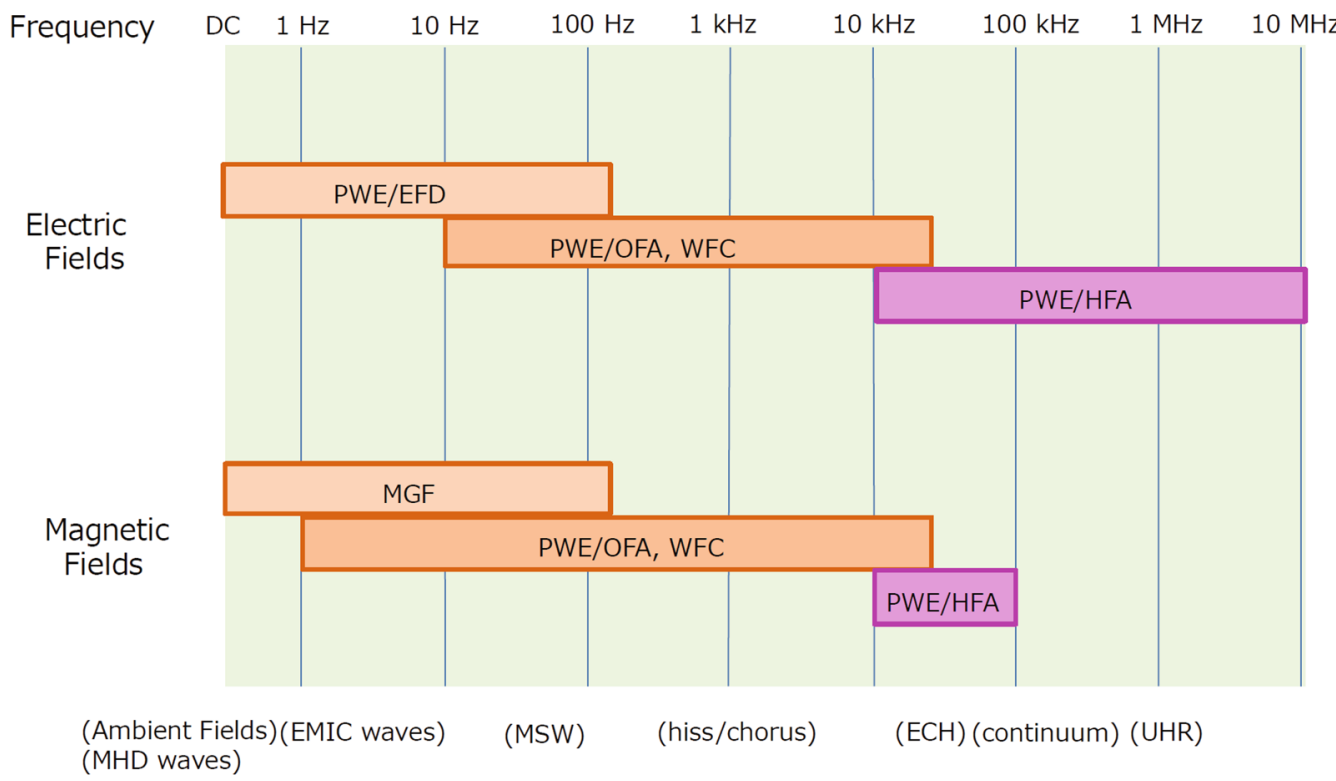

(AKR, radio emissions)

Fig. 5 a Energy coverage of electron measurements from LEP-e, MEP-e, HEP, and XEP, and ion measurements from LEP-i and MEP-i. b Frequency coverage of electric field and magnetic field measurements from PWE (EFD, OFA/WFC, HFA) and MGF

generation of plasma waves through anisotropies in the distribution function, and the LEP-e/MEP-e measurements identify the variations in the distribution function associated with the wave-particle interactions.
The LEP-i and MEP-i measure ions from $10 \mathrm{eV} / \mathrm{q}$ to $180 \mathrm{keV} / \mathrm{q}$ with mass discrimination. Ion species are discriminated using the time-of-flight method. These ion observational data are used to identify the free energy source to generate EMIC waves and magnetosonic mode 
waves (equatorial noise) (e.g., Kasahara et al. 1994; Ma et al. 2014). The data are also used to study the ring current evolution and variations of ion compositions, which are necessary for understanding how ion distributions modify the magnetic field configuration during geospace storms (e.g., Nose et al. 2005).

The plasma wave experiment (PWE) observes electric fields in the frequency range from $\mathrm{DC}$ to $10 \mathrm{MHz}$ and magnetic fields in the frequency range from a few $\mathrm{Hz}$ to $100 \mathrm{kHz}$ (Kasahara et al. 2018c). The electric field component is measured by two pairs of wire dipole antennas with a tip-to-tip length of $30 \mathrm{~m}$, and the magnetic field component is measured using three-axis search coils (Ozaki et al. 2018). The PWE consists of four subcomponents: an electric field detector (EFD) (Kasaba et al. 2017), onboard frequency analyzer (OFA), waveform capture (WFC) (Kasahara et al. 2018c; Matsuda et al. 2018), and high-frequency analyzer (HFA) (Kumamoto et al. 2018). The EFD produces low-frequency waveform data with a sampling frequency of $512 \mathrm{~Hz}$ and the spacecraft potential. The EFD measures the ambient electric fields and ULF waves as well as the EMIC waves. The OFA produces the power spectrum as well as the spectrum matrix for both electric fields and magnetic fields from $10 \mathrm{~Hz}$ to $20 \mathrm{kHz}$. The time resolutions of the power spectrum and spectrum matrix are nominally 1 and $8 \mathrm{~s}$, respectively, while the possible time resolutions are $0.5-4$ and 4-32 s, respectively. The WFC observes the raw waveforms of the electric/magnetic fields with $65-\mathrm{kHz}$ sampling for the chorus burst mode and $1-\mathrm{kHz}$ sampling for the EMIC burst mode. As the data size of the WFC is often too large to download to the ground without any data reduction, the OFA data are also used to select the WFC data period for downloading to the ground. The HFA produces the power spectra for the electric fields $(10 \mathrm{kHz}-10 \mathrm{MHz})$ and magnetic fields $(10-100 \mathrm{kHz})$, and the data are used to measure the upper hybrid resonance (UHR) waves and several radio waves such as auroral kilometric radiation (AKR) and type III solar radio bursts. Because the UHR frequency is a function of the ambient electron density, the HFA provides the ambient plasma density along the orbit.

The magnetic field experiment (MGF) measures magnetic fields from $\mathrm{DC}$ to several tens of $\mathrm{Hz}$ (the sampling frequency is $256 \mathrm{~Hz}$ ). That is, the MGF observes the ambient magnetic fields, i.e., the background field strength and direction, ULF waves, and EMIC waves (Matsuoka et al. 2018). The field direction error of the MGF measurements is less than $1^{\circ}$, so it is possible to analyze the field distortions in detail due to the ring current and field-aligned current.

To understand energy exchange processes between electrons and waves through wave-particle interactions, a software-type wave-particle interaction analyzer (S-WPIA) is installed on the satellite (Katoh et al. 2018; Hikishima et al. 2018). The phase difference between the electric/magnetic fields of the plasma waves and gyrating electrons determines whether the waves accelerate the electrons, or the electrons generate the waves. The S-WPIA system calculates the phase difference with a time resolution of $10 \mu$ s under the cooperation of the MEP-e/HEP/XEP, PWE, and MGF instruments. The S-WPIA realizes a new observation to identify unambiguously how energy conversion occurs in the wave-particle interactions.

The ERG satellite provides not only the data mentioned above but also quasi-real time space weather data. The space weather data include datasets from the XEP, HEP, and MGF instruments. These data are downloaded to the ground immediately after the observation and are displayed on the webpage of JAXA. These data are not fully calibrated, so it is not always possible to use the quantitative analyses, but the data are useful for monitoring the current space environment.

\section{Ground-network observations and integrated data analysis/simulation}

In addition to the ERG satellite observations, the project consists of the ground-network observations and integrated data analysis/simulation teams. Conjugate observations between the ERG satellite and multi-point ground-based network are important for a comprehensive understanding of geospace because the groundbased network observations provide the data at different MLTs and invariant latitudes (Shiokawa et al. 2017). For example, Super Dual Aurora Radar Network (SuperDARN) radars provide the data for convection electric fields and ULF pulsations at different MLTs and latitudes. Magnetometer networks provide temporal-spatial distributions of EMIC (Pc1) and Pc3-Pc5 ULF waves as well as ionospheric currents. Very low frequency (VLF)/ extremely low frequency (ELF) receivers observe the chorus/hiss waves. The optical imagers observe electron and proton auroras, which indicate precipitations of electrons and ions from a few hundred $\mathrm{eV}$ to a few tens of $\mathrm{keV}$. Riometers observe cosmic noise absorption, which is a manifestation of tens of $\mathrm{keV}$ electron precipitations. The standard radio wave observations provide information about disturbances in the D-layer, which are proxies for sub-relativistic/relativistic electron precipitations. These networks of optical imagers, riometers, and standard radio wave observations can be used to specify the spatial location and extent of precipitating particles and waves. The incoherent scatter radar such as European Incoherent Scatter Radar (EISCAT) provides the energy spectrum of precipitating electrons. 
In order to investigate the physical processes quantitatively and reveal the relative role of each process, integrated data analyses with the numerical simulations are indispensable (Seki et al. 2018). The multi-point observations of the ERG satellite and ground-based observations provide a macroscopic view of geospace, which can be compared to the global simulation for the inner magnetosphere, e.g., the evolution of the plasmasphere, ring current, and radiation belts. In contrast, microscopic wave-particle interactions can also be compared with the detailed simulations of the wave-particle interactions (e.g., Katoh and Omura 2007; Hikishima et al. 2009; Saito et al. 2012).

\section{ERG science center}

The raw data acquired from the satellite are archived at ISAS. After the data evaluation and necessary processing to generate level-1 data by the instrument teams, level-2 data are generated at the ERG science center (Miyoshi et al. 2018). Level-3 data are generated by combining with the data from several instruments. For example, the electron density along the satellite orbit is level-3 data, which are generated from the UHR frequency measured by the PWE/HFA and the electron gyrofrequency measured by the MGF. Level-4 data are the phase space densities of electrons and ions, which are generated with the inter-calibrated data between different particle instruments. All science data files are distributed from the ERG science center.

In order to realize the integrated data analyses using not only the ERG satellite data but also the ground-based data, other satellite data, and simulation data, the data format and data analysis tools are important. All observation data from the ERG project are archived in the Common Data Format (CDF) that is a common format for the space physics community. The Space Physics Environment Data Analysis System (SPEDAS) (Angelopoulos 2008) is used for the data analysis software in the ERG project, which involves several Interactive Data Language (IDL) programs and is commonly used in the space physics community. The ERG science center developed the SPEDAS plug-in programs for the ERG project, and users can download and manipulate the CDF files of the ERG project using the plug-in programs. Because SPEDAS includes many plug-in programs for not only the ERG project but also other satellites and ground-based observations, users can readily access and analyze different kinds of datasets.

\section{Initial observations}

Here, we present the initial observation results from the ERG satellite. Note that the electric field intensity from the PWE instrument in this paper is calculated under the assumption of theoretical antenna capacitance in vacuum to the impedance of the electric field sensors (Kasahara et al. 2018c). Figure 6 shows a summary plot for all instrumental data from the ERG satellite on April 9, 2017. Moderate geomagnetic activity is recorded. The minimum Dst index on this day is $-25 \mathrm{nT}$. As shown in panel (m), a series of substorms are observed before 17:00 UT, and then, the geomagnetic activity decreases. During this period, the apogee MLT of the satellite is approximately 04:00 MLT.

Panels (a), (b), (c), and (d) present frequency-time diagrams of the PWE/HFA, PWE/OFA (electric fields), PWE/OFA (magnetic fields), and PWE/EFD, respectively. These instruments cover the frequency ranges from DC to $10 \mathrm{MHz}$. The two lines in panels (a), (b), and (c) indicate the local electron gyrofrequency and the half gyrofrequency derived from the MGF measurements.

In panel (a), emissions of several kinds of plasma waves and radio waves are observed. AKR is observed at a few hundred $\mathrm{kHz}$, and the UHR emissions above the electron gyrofrequency are almost always observed along the orbit. As the PWE/HFA covers wide frequency up to $10 \mathrm{MHz}$, the satellite observes the UHR emissions during the whole orbit. Using the frequency of the UHR emissions, it is possible to estimate the electron density from the magnetosphere to the topside ionosphere.

In panels (b) and (c), chorus waves at a few hundred $\mathrm{Hz}$ to a few $\mathrm{kHz}$ are detected outside the plasmapause where the ambient density is low, and both lower-band and upper-band chorus waves are sometimes clearly observed. There are several periods when the frequency of chorus emissions is relatively low compared to the local half gyrofrequency because the satellite traverses off the equatorial plane. Inside the plasmapause, whistler mode hiss waves are observed. Panel (d) presents a frequency-time diagram of the low-frequency electric fields covering the ULF and ELF ranges. Several electrostatic waves are observed, and the banded emissions at $70 \mathrm{~Hz}$ at about 23:00 UT are magnetosonic mode waves (equatorial noise).

Panels (e), (f), (g), and (h) present energy-time diagrams of the XEP, HEP, MEP-e, and LEP-e instruments. Note that the color bar indicates the count for the XEP, HEP, and LEP-e, and the flux for the MEP-e. Wideenergy electron measurements are achieved from $17 \mathrm{eV}$ to $20 \mathrm{MeV}$. The energy-time diagram of the XEP shows the outer radiation belt population. The peak of the count is $L \sim 4.5$, and a clear inner boundary of the outer radiation belt is observed at $L \sim 3.3$ in every path. Note that the $L$-value in this paper is the Mcllwain L (Mcllwain 1961) derived from the International Geomagnetic Reference Field model. 


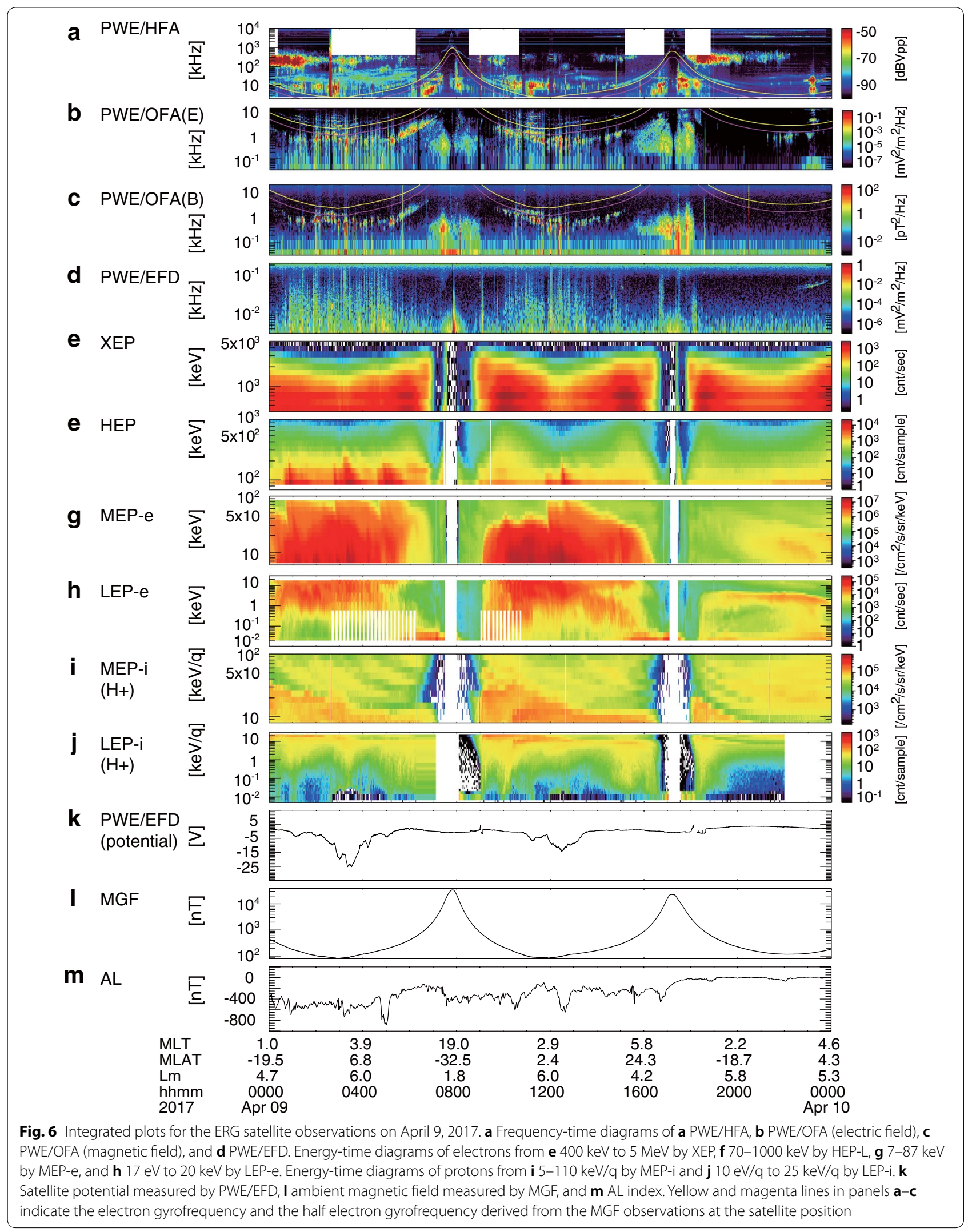


The LEP-e, MEP-e, and HEP clearly detect dynamical variations of electrons from a few hundred $\mathrm{eV}$ to a few tens of $\mathrm{keV}$ inside the outer radiation belt. The white blank periods for the LEP-e measurements in panel (h) correspond to the loss-cone measurements (Kazama et al. 2017). During this period, the satellite performs conjugate observations with ground instruments, and the LEP-e instruments measure the electron flux variations inside the loss cone to identify the precipitation of auroral electrons. Sometimes, these three instruments observe signatures of injections in which energy dispersions are observed from a few $\mathrm{keV}$ to a few hundred $\mathrm{keV}$ electrons. It is expected that electrons injected from the night side drift to the morning side where the satellite observes them.

Panels (i) and (j) present energy-time spectrograms of protons by the MEP-i and LEP-i. Note that the color bar indicates the count for the LEP-i and the flux for the MEP-i. As the satellite is on the dawn side, the flux of ions is relatively small, but energy-dependent structures are clearly detected. Inside the plasmapause, low-energy ions below $1 \mathrm{keV}$ are detected.

Panel $(\mathrm{k})$ shows the time variations of the satellite potential measured with the PWE/EFD. The negative charging of the satellite is observed. Panel (l) shows the magnetic field observed by the MGF. The intensity of the magnetic field is less than $100 \mathrm{nT}$ near the apogee, while the intensity is larger than $35,000 \mathrm{nT}$ near the perigee. During this period, the MGF measures variations in the magnetic field along the orbit, e.g., deformations of the magnetic field and ULF pulsations.

As shown in panel (m), several substorm activities are observed during this period. Associated with the substorm activities from 00:00 to 14:00 UT, injections of electrons are detected with the LEP-e, MEP-e, and HEP. As seen in panels (b) and (c), intense chorus emissions are associated with the injections. Moreover, the potential clearly decreases, as shown in panel $(\mathrm{k})$, during the injections of hot electrons and sub-relativistic electrons, indicating satellite charging during this period.

After 17:00 UT, the geomagnetic activities decrease and no substorms are observed. Relativistic electrons of the outer radiation belt do not change significantly compared with previous periods, as shown by the XEP and HEP measurements [panels (e) and (f), respectively]. In contrast, the low-energy electron fluxes decrease, as shown in the MEP-e and LEP-e measurements. Especially, the tens of $\mathrm{keV}$ electrons measured with the MEP-e do not exhibit any significant variations during this period. Chorus waves are not observed in panels (b) and (c) because of the absence of free energy to generate chorus waves.

Figure 7 shows examples for various kinds of plasma waves observed by the PWE/OFA, PWE/WFC, PWE/
EFD, and MGF at different MLTs and radial distances. As shown in Fig. 7, the ERG satellite often observes whistler mode chorus waves outside the plasmapause, while the ERG satellite observes whistler mode hiss waves inside the plasmapause. Magnetosonic mode waves (equatorial noise) and EMIC waves are observed inside and outside the plasmapause. Waveforms of Pc4 and Pc5 pulsations observed by the MGF are shown. Both PWE/EFD and MGF always measure waveform of Pc4 and Pc5 pulsations and EMIC waves, while PWE/WFC measures waveform for whistler mode waves and magnetosonic mode waves intermittently as burst observations. Since the operation time of PWE/WFC is limited due to the data recorder volume and the downlink rate to the ground (Takashima et al. 2018; Miyoshi et al. 2018), the burst observations are scheduled by considering the possible generation regions of waves and periods for conjugate observations with the ground-based observations and other satellites such as Van Allen Probes.

Conjugate burst observations between the ERG satellite and Van Allen Probes have been operated since June, 2017, and whistler mode chorus and hiss waves as well as lightning whistlers were simultaneously observed by both satellites at almost same $L$-shell but different magnetic latitudes. The ERG satellite operates burst observations to obtain waveform continuously with a $65-\mathrm{kHz}$ sampling and the maximum duration of $40 \mathrm{~min}$. From these observations, it becomes possible to investigate temporal and spatial variations of fine structure of the frequency spectrum of not only whistler mode chorus waves outside the plasmapause but also long duration whistler mode hiss waves inside the plasmapause. Temporal and spatial evolutions of these waves have been studied using these conjugate data. Further conjugate burst observations between the ERG satellite and other satellites will be planned to provide detailed information of generation and propagations of plasma waves in the inner magnetosphere.

Conjugate observations between the ERG satellite and ground-based network stations have been also operated. Simultaneous ground-satellite observations with several ground-based instruments such as high-speed optical cameras, VLF antennas, and the EISCAT radar have been realized. From the conjugate observation on March 27, 2017, one-to-one correspondences were identified among temporal variations of whistler mode chorus bursts, flux modulations of tens $\mathrm{keV}$ electrons inside the loss cone from the ERG satellite observation, and luminosity of the pulsating aurora from the ground-based observation, which is a direct evidence that the pitch angle scattering by whistler mode chorus bursts causes luminosity modulations of the pulsating aurora (Kasahara et al. 2018b). Since the ground-network observations provide the data 


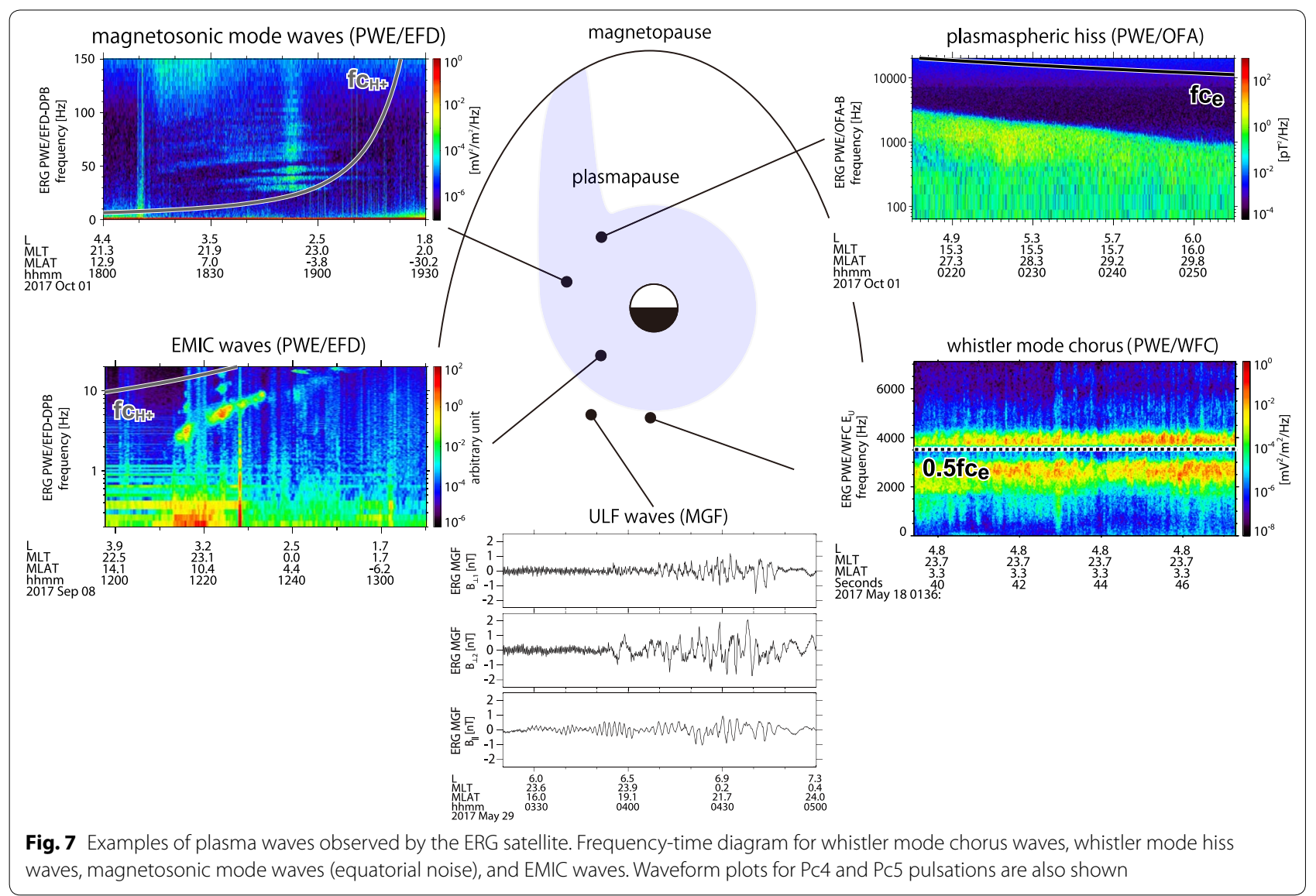

at different MLTs and the invariant latitudes (Shiokawa et al. 2017), these observations are complementary to the satellite observations and important to understand global phenomena in the inner magnetosphere. We will plan coordinated observations to take advantage of the most of merits of multi-point observations.

\section{Summary}

The ERG project was designed for understanding the energetic electron variations of the radiation belts and geospace storms. The ERG satellite was launched in December 2016 and started full-operation observations on March 24, 2017.

During solar cycle 24, several geospace satellites operate in the inner magnetosphere. The NASA Van Allen Probes explore the radiation belts and the inner magnetosphere. The NASA THEMIS (Angelopoulos 2008) and MMS (Burch et al. 2016) observe the magnetotail as well as the dayside magnetosphere. In addition, Geotail, Cluster, and other satellites operate in geospace. The ERG satellite has joined the international fleet of satellites and collaborated with them as well as with groundbased observations. The ERG project contributes to the comprehensive understanding of geospace with the international collaborations.

The ERG satellite has begun observations. The ERG project will lead to significant advances in the identification of the physical processes causing variations in the radiation belts and geospace storms, and in providing new insight into the cross-energy and cross-regional coupling processes.

\section{Authors' contributions}

YM is the project scientist of the ERG project and the lead author of this article. IS is the project manager. TT is the mission manager. KA is the leader of the mission team and PI of LEP-i. NH is the PI of XEP. TM is the PI of HEP. SK is the PI of MEP-e. SY is the PI of MEP-i. SYW is the project manager, SWYT is the project scientist, and Y. Kazama is the project engineer, and PTPH is the PI of LEP-e. Y. Kasahara is the PI of PWE. Y. Kasaba and SY are co-PI of PWE. AM is the PI of MGF. HK is the PI of SWPIA and Co-PI of PWE. Y. Katoh is the co-PI of SWPIA. K. Shiokawa is the PI of the ERG-ground network observations team. K. Seki is the PI of the integrated data analysis/simulation team. All authors read and approved the final manuscript.

\section{Author details}

${ }^{1}$ ISEE, Nagoya University, Nagoya 464-8601, Japan. ${ }^{2}$ ISAS, JAXA, Sagamihara 252-0222, Japan. ${ }^{3}$ RDD, JAXA, Tsukuba 305-8505, Japan. ${ }^{4}$ The University of Tokyo, Tokyo 113-8654, Japan. ${ }^{5}$ Osaka University, Toyonaka 560-0043, Japan. ${ }^{6}$ ASIAA, Taipei 10617, Taiwan, ROC. ${ }^{7}$ National Cheng Kung University, Tainan 70101, Taiwan, ROC. ${ }^{8}$ Kanazawa University, Kanazawa 920-1192, Japan. ${ }^{9}$ Tohoku University, Sendai 980-8578, Japan. ${ }^{10}$ RISH, Kyoto University, Uji 611-0011, Japan. 


\begin{abstract}
Acknowledgements
We thank all members of the ERG-project to their great efforts to realize this mission for many years. Prof. Takayuki Ono was the PI of the ERG project, and he passed away in December, 2013. We acknowledge his great efforts to drive this project. We also thank S. Matsuda and S. Kurita for their help to prepare the manuscript. A part of this work was supported by JSPS Grants-in-Aid for Scientific Research (23224011, 15H05747, 15H05815, 16H04056, 16 H01172, 16H06286, 16H02229). Data are distributed through the ERG Science Center operated by ISAS/JAXA and ISEE/Nagoya University.
\end{abstract}

\section{Competing interests}

The authors declare that they have no competing interests.

\section{Availability of data and materials}

All science data of the ERG project are distributed by the ERG Science Center (https://ergsc.isee.nagoya-u.ac.jp/index.shtml.en). The AL index is provided from WDC-C2, Kyoto University (http://wdc.kugi.kyoto-u.ac.jp/aedir/).

\section{Ethics approval and consent to participate}

Not applicable.

\section{Funding}

Apart of this work was supported by JSPS Grants-in-Aid for Scientific Research (23224011, 15H05747, 15H05815, and 16H06286, 16H02229). This study was supported by JSPS Bilateral Open Partnership Joint Research Projects. The ERG Science Center is operated by ISAS/JAXA and ISEE/Nagoya University. The development and operation of LEP-e is partly funded by Academia Sinica and National Cheng Kung University of Taiwan and also through the support of Ministry of Science and Technology of Taiwan under contract 106-2111-M001-011 and 105-3111-Y-001-042

\section{Publisher's Note}

Springer Nature remains neutral with regard to jurisdictional claims in published maps and institutional affiliations.

\section{Received: 16 April 2018 Accepted: 15 May 2018}

Published online: 26 June 2018

\section{References}

Angelopoulos V (2008) The THEMIS mission. Space Sci Rev. https://doi. org/10.1007/s11214-008-336-1

Asamura K, Kazama Y, Yokota S, Kasahara S, Miyoshi Y (2018) Low-energy particle experiments-ion mass analyzer (LEPi) onboard the ERG (Arase) satellite. Earth Planets Space 70:70. https://doi.org/10.1186/s4062 3-018-0846-0

Burch JL, Moore TE, Torbert RB, Giles BL (2016) Magnetospheric multiscale overview and science objectives. Space Sci Rev. https://doi.org/10.1007/ s11214-015-064-9

Ebihara Y, Miyoshi Y (2011) Dynamic inner magnetosphere: a tutorial and recent advances. In: Liu W, Fujimoto M (eds) The dynamic magnetosphere. Springer, Berlin. https://doi.org/10.1007/978-94-007-0501-2_9

Elkington SR, Hudson MK, Chan AA (1999) Acceleration of relativistic electrons via drift-resonant interaction with toroidal-mode Pc-5 ULF oscillations. Geophys Res Lett 26:3273-3276

Hikishima M, Yagitani S, Omura Y, Nagano I (2009) Full particle simulation of whistler mode rising chorus emissions in the magnetosphere. J Geophys Res. https://doi.org/10.1029/2008ja013625

Hikishima M, Kojima H, Katoh Y, Kasahara Y, Kasahara S, Mitani T, Higashio N, Matsuoka A, Miyoshi Y, Asamura K, Takashima T, Yokota S, Kitahara M, Matsuda S (2018) Data processing in software-type wave-particle interaction analyzer on board the Arase satellite. Earth Planets Space 70:80. https:// doi.org/10.1186/s40623-018-0856-y

Kasaba Y, Ishisaka K, Kasahara Y, Imachi T, Yagitani S, Kojima H, Matsuda S, Shoji M, Kurita S, Hori T, Shinbori A, Teramoto M, Miyoshi Y, Nakagawa T, Takahashi N, Nishimura Y, Matsuoka A, Kumamoto A, Tsuciya F, Nomura $R$ (2017) Wire probe antenna (WPT) and electric field detector (EFD) of plasma wave experiment (PWE) aboard the Arase satellite: specifications and initial evaluation results. Earth Planets Space 69:174. https://doi. org/10.1186/s40623-017-0760-X

Kasahara Y, Kenmochi H, Kimura I (1994) Propagation characteristics of the ELF emissions observed by the satellite Akebono in the magnetic equatorial region. Radio Sci. https://doi.org/10.1029/94rs00445

Kasahara S, Yokota S, Mitani T, Asamura K, Hirahara M, Shibano Y, Takshima $\mathrm{T}$ (2018a) Medium-energy particle experiments-electron analyzer (MEP-e) for the exploration of energization and radiation in geospace (ERG) mission. Earth Planets Space 70:69. https://doi.org/10.1186/s4062 3-018-0847-z

Kasahara S, Miyoshi Y, Yokota S, Mitani T, Kasahara Y, Matsuda S, Kumamoto A, Matsuoka A, Kazama Y, Frey HU, Angelopoulos V, Kurita S, Keika K, Seki K, Shinohara I (2018b) Pulsating aurora from electron scattering by chorus waves. Nature 556:7692. https://doi.org/10.1038/nature25505

Kasahara Y, Kasaba Y, Kojima H, Yagitani S, Ishisaka K, Kumamoto A, Tsuchiya F, Ozaki M, Matsuda S, Imachi T, Miyoshi Y, Hikishima M, Katoh Y, Ota M, Shoji M, Matsuoka A, Shinohara I (2018c) The plasma wave experiment (PWE) on board the Arase (ERG) satellite. Earth Planets Space 70:86. https ://doi.org/10.1186/s40623-018-0842-4

Katoh Y, Omura Y (2007) Computer simulation of chorus wave generation in the Earth's inner magnetosphere. Geophys Res Lett. https://doi. org/10.1029/2006gl028594

Katoh Y, Kojima H, Hikishima M, Takazhima T, Asamura K, Miyoshi Y, Kasahara S, Mitani T, Higashio N, Matsuoka A, Ozaki M, Yagitani S, Yokota S, Matsuda S, Kitahara M, Shinohara I (2018) Software-type wave-particle interaction analyzer on board the Arase satellite. Earth Planets Space 70:4. https://doi. org/10.1186/s40623-017-0771-7

Kazama Y, Wang BJ, Wang SY, Ho PTP, Tam SWY, Chang TF, Chiang CY, Asamura K (2017) Low-energy particle experiments-electron analyzer (LEPe) onboard the Arase spacecraft. Earth Planets Space 69:165. https://doi. org/10.1186/s40623-017-0748-6

Kumamoto A, Tsuchiya F, Kasahara Y, Kasaba Y, Kojima H, Yagitani S, Ishisaka K, Imachi T, Ozaki M, Matsuda S, Shoji M, Matsuoka A, Katoh Y, Miyoshi Y, Obara T (2018) High frequency analyzer (HFA) of plasma wave experiment (PWE) onboard the Arase spacecraft. Earth Planets Space 70:82. https://doi.org/10.1186/s40623-018-0854-0

Ma Q, Li W, Chen L, Thorne RM, Angelopoulos V (2014) Magnetosonic wave excitation by ion ring distribution in the Earth's magnetosphere. J Geophys Res. https://doi.org/10.1002/2014gl061414

Matsuda S, Kasahara Y, Kojima H, Kasaba Y, Yagitani S, Ozaki M, Imachi T, Ishisaka K, Kumamoto A, Tsuchiya F, Ota M, Kurita S, Miyoshi Y, Hikishima M, Matsuoka A, Shinohara I (2018) Onboard software of plasma wave experiment aboard Arase: instrument management and signal processing of wave form capture/onboard frequency analyzer. Earth Planets Space 70:75. https://doi.org/10.1186/s40623-018-0838-0

Matsuoka A, Teramoto M, Nomura R, Nose M, Fujimoto A, Tanaka Y, Shinohara M, Nagatsuma T, Shiokawa K, Obana Y, Miyoshi Y, Mita M, Takashima T, Shinohara I (2018) The Arase (ERG) magnetic field investigation. Earth Planets Space 70:43. https://doi.org/10.1186/s40623-018-0800-1

Mauk BH, Fox NJ, Kanekal SG, Kessel RL, Sibeck DG, Ukhorskiy A (2013) Science objectives and rationale for the radiation belt storm probes mission. Space Sci Rev. https://doi.org/10.1007/s11214-012-9908-y

Mcllwain CE (1961) Coordinates for mapping the distribution of magnetically trapped particles. J Geophys Res. https://doi.org/10.1029/jz066i011p 03681

Mitani T, Takashima T, Kasahara S, Miyake W, Hirahara M (2018) High-energy electron experiments (HEP) aboard the ERG (Arase) satellite. Earth Planets Space 70:77. https://doi.org/10.1186/s40623-018-0853-1

Miyoshi Y, Kataoka R (2005) Ring current ions and radiation belt electrons during geomagnetic storms driven by coronal mass ejections and corotating interaction regions. Geophys Res Lett. https://doi.org/10.1029/2005G L024590

Miyoshi Y, Morioka A, Obara T, Misawa H, Nagai T, Kasahara Y (2003) Rebuilding process of the outer radiation belt during the November 3: 1993, magnetic storm-NOAA and EXOS-D observations. J Geophys Res. https ://doi.org/10.1029/2001ja007542

Miyoshi Y, Sakaguchi K, Shiokawa K, Evans D, Albert J, Conners M, Jordanova V (2008) Precipitation of radiation belt electrons by EMIC waves, observed from ground and space. Geophys Res Lett. https://doi.org/10.1029/2008G L035727 
Miyoshi Y, Ono T, Takashima T, Asamura K, Hirahara M, Kasaba Y, Matsuoka A, Kojima H, Shiokawa K, Seki K, Fujimoto M, Nagatsuma T, Cheng CZ, Kazama Y, Kasahara S, Mitani T, Matsumoto H, Higashio N, Kumamoto A, Yagitani S, Kasahara Y, Ishisaka K, Blomberg L, Fujimoto A, Katoh Y, Ebihara Y, Omura Y, Nose Hori T, Miyashita Y, Tanaka Y-M, Segawa, ERG working group (2012) The energization and radiation in geospace (ERG) project. In: Summers D, Mann IR, Baker DN, Schulz M (eds) Dynamics of the Earth's radiation belts and inner magnetosphere. American Geophysical Union, Washington. https://doi.org/10.1029/2012gm001304

Miyoshi Y, Kataoka R, Ebihara Y (2016) Flux enhancement of relativistic electrons associated with substorms. In: Balasis G, Daglis IA, Mann IR (eds) Waves, particles, and storms in geospace. Oxford Press, Oxford, pp 333-353

Miyoshi Y, Hori T, Shoji M, Teramoto M, Chang T-F, Segawa T, Umemura N, Matsuda S, Kurita S, Keika K, Miyashita Y, Seki K, Tanaka Y, Nishitani N, Kasahara S, Yokota S, Matsuoka A, Kasahara Y, Asamura K, Takashima T, Shinohara I (2018) The ERG science center. Earth Planets Space 70:96. https://doi. org/10.1186/s40623-018-0867-8

Nagai T (1988) Space weather forecast: prediction of relativistic electron intensity at synchronous orbit. Geophys Res Lett 15:425

Nakamura Y et al (2018) Exploration of energization and radiation in geospace (ERG): challenges, development, and operation of satellite system. Earth Planets Space. https://doi.org/10.1186/s40623-018-0863-z

Nose $\mathrm{M}$ et al (2005) Overwhelming $\mathrm{O}^{+}$contribution to the plasma sheet energy density during the October 2003 superstorm: geotail/EPIC and IMAGE/LENA observations. J Geophys Res 110:A09S24. https://doi. org/10.1029/2004ja010930

Omura Y, Hikishima Katoh Y, Summers D, Yagitani S (2009) Nonlinear mechanisms of lower-band and upper-band VLF chorus emissions in the inner magnetosphere. J Geophys Res. https://doi.org/10.1029/2009JA014206

Ozaki M, Yagitani S, Kojima H, Kasahara Y, Kasaba Y, Matsuoka A, Sasaki T, Yumoto T (2018) Magnetic search coil (MSC) of plasma wave experiment (PWE) aboard the Arase (ERG) satellite. Earth Planets Space 70:76. https:// doi.org/10.1186/s40623-018-0837-1

Reeves GD, McAdams KL, Friedel RHW, O'Brien TP (2003) Acceleration and loss of relativistic electrons during geomagnetic storms. Res Lett, Geophys. https://doi.org/10.1029/2002GL016513

Reeves GD, Spence HE, Henderson MG, Morely SK, Friedel RHW, Funsten HO, Baker DN, Kanekal SG, Blake JB, Fennell JF, Claudepierre SG, Thorne RM, Turner DL, Kletzing CA, Kurth WS, Larson BA, Niehof JT (2013) Electron acceleration in the heart of the Van Allen radiation belts. Science. https:// doi.org/10.1126/science.1237743
Saito S, Miyoshi Y, Seki K (2012) Relativistic electron microbursts associated with whistler chorus rising tone elements: GEMSIS-RBW simulations. J Geophys Res. https://doi.org/10.1029/2012JA018020

Seki K, Miyoshi Y, Ebihara Y, Katoh Y, Amano T, Saito S, Shoji M, Nakamizo A, Keika K, Hori T, Nakano S, Watanabe S, Kamiya K, Takahashi N, Omura Y, Nose M, Fok MC, Tanaka T, leda A, Yoshikawa A (2018) Theory, modeling, and integrated studies in the Arase (ERG) project. Earth Planets Space 70:17. https://doi.org/10.1186/s40623-018-0785-9

Shiokawa K, Katoh Y, Hamaguchi Y, Yamamoto Y, Adachi T, Ozaki M, Oyama SI, Nose M, Nagatsuma T, Tanaka Y, Otsuka Y, Miyoshi Y, Kataoka R, Takagi Y, Takeshita Y, Shinbori A, Kurita S, Hori T, Nishitani N, Shinohara I, Tsuchiya F, Obana Y, Suzuki S, Takahashi N, Seki K, Kadokura A, Hosokawa K, Ogawa Y, Connors M, Ruohoniemi JM, Engebretson M, Turunen E, Ulich T, Manninen J, Kurkin V, Oinats A, Pashinin A, Vasilyev R, Rakhmatulin R, Bristow W, Karjala M (2017) Ground-based instruments of the PWING project to investigate dynamics of the inner magnetosphere as subauroral latitudes as a part of the ERG-ground coordinated observation network. Earth Planets Space 69:160. https://doi.org/10.1186/s40623-017-0745-9

Shprits YY, Elkington SR, Meredith NP, Subbotin DA (2008a) Review of modeling of losses and sources of relativistic electrons in the outer radiation belt I: radial transport. J Atm Solar Terr Phys 70:1679-1693

Shprits YY, Subbotin DA, Meredith NP, Elkington SR (2008b) Review of modeling of losses and sources of relativistic electrons in the outer radiation belt II: local acceleration and loss. J Atmos Solar Terr Phys 70:1694-1713

Summers D, Thorne RM, Xiao F (1998) Relativistic theory of wave-particle resonant diffusion with application to electron acceleration in the magnetosphere. J Geophys Res 103:20487-20500

Takashima T, Ogawa E, Asamura K, Hikishima M (2018) Design of mission network system with SpaceWire for scientific payloads onboard the Arase spacecraft. Earth Planet Space, https://doi.org/10.1186/s4062 3-018-0839-z

Thorne RM (2010) Radiation belt electrons: The importance of wave-particle interactions. Res Lett, Geophys. https://doi.org/10.1029/2010GL044990

Turner DL, Morley SK, Miyoshi Y, Ni B, Huang C-L (2012) Outer belt flux dropouts: current understanding and unresolved questions. In: Summers D, Mann IR, Baker DN, Schulz M (eds) Dynamics of the Earth's radiation belts and inner magnetosphere. American Geophysical Union, Washington. https://doi.org/10.1029/2012gm001310

Yokota S, Kasahara S, Mitani T, Asamura K, Hirahara M, Takashima T, Yamamoto K, Shibano Y (2017) Medium-energy particle experiments-ion mass analyzer (MEP-i) onboard ERG (Arase). Earth Planets Space 69:172. https:// doi.org/10.1186/s40623-017-0754-8

\section{Submit your manuscript to a SpringerOpen ${ }^{\circ}$ journal and benefit from:}

- Convenient online submission

- Rigorous peer review

- Open access: articles freely available online

- High visibility within the field

- Retaining the copyright to your article

Submit your next manuscript at springeropen.com 